\title{
Genome-wide transcription start site profiling in biofilm-grown Burkholderia cenocepacia J2315
}

\author{
Andrea M. Sass ${ }^{1 *}$, Heleen Van Acker ${ }^{1}$, Konrad U. Förstner ${ }^{3}$, Filip Van Nieuwerburgh ${ }^{2}$, Dieter Deforce ${ }^{2}$, \\ Jörg Vogel ${ }^{4}$ and Tom Coenye ${ }^{1}$
}

\begin{abstract}
Background: Burkholderia cenocepacia is a soil-dwelling Gram-negative Betaproteobacterium with an important role as opportunistic pathogen in humans. Infections with B. cenocepacia are very difficult to treat due to their high intrinsic resistance to most antibiotics. Biofilm formation further adds to their antibiotic resistance. B. cenocepacia harbours a large, multi-replicon genome with a high GC-content, the reference genome of strain 12315 includes 7374 annotated genes. This study aims to annotate transcription start sites and identify novel transcripts on a whole genome scale.

Methods: RNA extracted from B. cenocepacia J2315 biofilms was analysed by differential RNA-sequencing and the resulting dataset compared to data derived from conventional, global RNA-sequencing. Transcription start sites were annotated and further analysed according to their position relative to annotated genes.

Results: Four thousand ten transcription start sites were mapped over the whole B. cenocepacia genome and the primary transcription start site of 2089 genes expressed in B. cenocepacia biofilms were defined. For 64 genes a start codon alternative to the annotated one was proposed. Substantial antisense transcription for 105 genes and two novel protein coding sequences were identified. The distribution of internal transcription start sites can be used to identify genomic islands in B. cenocepacia. A potassium pump strongly induced only under biofilm conditions was found and 15 non-coding small RNAs highly expressed in biofilms were discovered.
\end{abstract}

Conclusions: Mapping transcription start sites across the B. cenocepacia genome added relevant information to the J2315 annotation. Genes and novel regulatory RNAs putatively involved in B. cenocepacia biofilm formation were identified. These findings will help in understanding regulation of $B$. cenocepacia biofilm formation.

Keywords: Burkholderia cenocepacia, Biofilms, dRNA-Seq, Transcription start site, Small RNAs, Antisense RNA, Genomic islands

\section{Background}

Burkholderia cenocepacia J2315 is a member of the Burkholderia cepacia complex (Bcc), a group of 18 species of closely related Gram-negative Betaproteobacteria [1] which occur in the soil rhizosphere and also play an important role as opportunistic pathogens in humans [2-4]. Bcc bacteria are intrinsically resistant to most antibiotics, and infections with Bcc bacteria are therefore difficult to treat. Bcc bacteria are also able to form biofilms, further adding to their recalcitrance to antibiotic treatment [4].

B. cenocepacia J2315 harbours a large $8.06 \mathrm{Mb}$ multireplicon genome with a high average GC-content of $66.9 \%$. The genome consists of two large replicons of

\footnotetext{
*Correspondence: Andrea.Sass@UGent.be

'Laboratory of Pharmaceutical Microbiology, Ghent University,

Ottergemsesteenweg 460, 9000 Ghent, Belgium

Full list of author information is available at the end of the article
}

3.87 and $3.22 \mathrm{Mb}$, a smaller replicon $0.88 \mathrm{Mb}$ and a plasmid $0.09 \mathrm{Mb}$ in size, with 7261 annotated protein coding and 113 annotated RNA genes [5], including 74 tRNAs and 10 riboswitches. However, transcription start sites (TSS), 5' untranslated regions (5'UTRs) of annotated genes and regulatory non-coding small RNAs have not yet been comprehensively analysed and annotated. Emerging new RNA sequencing techniques, notably differential RNA sequencing (dRNA-Seq, [6]), make it now possible to precisely map the transcription start sites over a whole genome, and at the same time discover novel genome features.

Primary transcripts of prokaryotes carry a triphosphate at their $5^{\prime}$-end, whereas $5^{\prime}$-ends derived from processing and degradation carry a monophosphate. The dRNA-Seq approach uses the properties of a $5^{\prime}$-monophosphatedependent exonuclease (Terminator ${ }^{\mathrm{mix}} 5^{\prime}$-PhosphateDependent Exonuclease, TEX) to selectively degrade 
processed transcripts, thereby enriching for un-processed RNA species carrying a native $5^{\prime}$-triphosphate. TSS can then be identified by comparing TEX-treated with untreated RNA-seq libraries, as they appear as localised maxima in coverage enriched by TEX-treatment [6].

dRNA-Seq enables precise mapping of $5^{\prime}$ ends of transcripts, whereas coverage over the whole transcript length is usually poor and 3' end of transcripts are only represented for short transcripts. For this reason the dRNA-Seq datasets were compared to conventional global RNA-seq data (gRNA-Seq) which provide more even coverage and a more comprehensive representation of full length transcription units. This approach aids in evaluating the function of an identified TSS, particularly for TSS internal to genes.

The aim of the present study is to identify genes expressed in B. cenocepacia biofilms and detect the regulatory elements that might be involved in biofilm formation and survival, as a prerequisite to develop new strategies in treatment of B. cenocepacia infections.

\section{Results and discussion TSS annotation}

dRNA-Seq of duplicate biofilm-derived RNA samples resulted in datasets with 2.4-4.1 million mapped reads, gRNA-Seq of triplicate biofilm-derived RNA samples resulted in datasets with 23-33 million mapped reads (Additional file 1: Table S1). A total of 10843 TSS were automatically annotated based on the dRNA-Seq data (Additional file 2: Table S2), evenly distributed on forward and reverse strands. 3908 TSS remained after noise filtering on a minimum of 10 read starts (Table 1, Additional file 3: Table S3). These were then categorised according to their position in relation to annotated genes (Fig. 1a): TSS in intergenic regions, located $\leq 300 \mathrm{nt}$ upstream of the start of and in sense with an annotated gene, were assigned primary TSS (pTSS) for the respective gene. TSS internal to annotated genes were assigned internal sense (isTSS) or antisense (asTSS). TSS in intergenic regions and not associated with any gene were assigned "orphan" (oTSS). Where TSS were positioned within $100 \mathrm{nt}$ of and same sense to a primary or orphan TSS, they were designated secondary (sTSS).

TSS loci were also compared to the global transcriptome datasets by manual inspection. More than $90 \%$ of intergenic pTSS were accompanied by an abrupt increase in coverage in the gRNA-Seq dataset (Fig. 1b), substantiating that they are bona fide loci for transcription initiation. For the purpose of differentiation from TSS based on dRNA-Seq data, we designate these abrupt increases in gRNA-Seq data coverage "leading edges of transcription" (LEs, [7]) for the rest of the manuscript.

LEs found internal to genes were used to assign function to internal TSS: where internal TSS were associated with LEs and positioned $\leq 300 \mathrm{nt}$ upstream or $\leq 100 \mathrm{nt}$ downstream and in sense with a gene lacking an intergenic pTSS, they were re-assigned pTSS for the respective gene (Fig. 1c).

Transcription can be primed by molecules other than NTPs, e.g. nano rRNAs [8]. The resulting primary transcripts do not carry a triphosphate at their 5 '-end and are depleted by TEX-treatment. Where depleted local read start maxima coincided with a distinct LE, they were reported as depleted pTSS (Table 1, Additional file 3: Table S3), adding another 102 pTSS to the dataset.

In total 2089 pTSS were annotated over the whole $B$. cenocepacia genome for genes transcribed under biofilm conditions, representing $28 \%$ of all annotated genes. This proportion appears realistic when comparing it to values found in similar studies (24\% for Salmonella enterica [9] and $51 \%$ for Helicobacter pylori [10]), and considering that the large $B$. cenocepacia genome consists of a high number of non-essential genes [11] that are not all transcribed in the one growth condition analysed in the present study. Most pTSS were located on the large replicon (Table 1), which is to be expected since the large replicon harbours most essential genes [11]. Intergenic pTSS

Table 1 Number of transcription start sites by category

\begin{tabular}{|c|c|c|c|c|c|}
\hline & Replicon 1 & Replicon 2 & Relicon 3 & Plasmid & Total \\
\hline Genes & 3622 & 2859 & 781 & 100 & 7374 \\
\hline TSS & 6815 & 3010 & 914 & 104 & 10843 \\
\hline Total categorised TSS & 2595 & 1142 & 316 & 57 & 4010 \\
\hline pTSS & 1271 & 671 & 136 & 11 & 2089 \\
\hline depleted pTSS & 64 & 24 & 8 & 6 & 102 \\
\hline pTSS internal same gene & 42 & 27 & 2 & 1 & 72 \\
\hline pTSS internal upstream gene & 78 & 22 & 5 & 0 & 105 \\
\hline oTSS & 237 & 126 & 57 & 8 & 428 \\
\hline sTSS & 181 & 61 & 19 & 0 & 261 \\
\hline isTSS & 502 & 140 & 55 & 15 & 712 \\
\hline asTSS & 304 & 144 & 49 & 23 & 520 \\
\hline
\end{tabular}




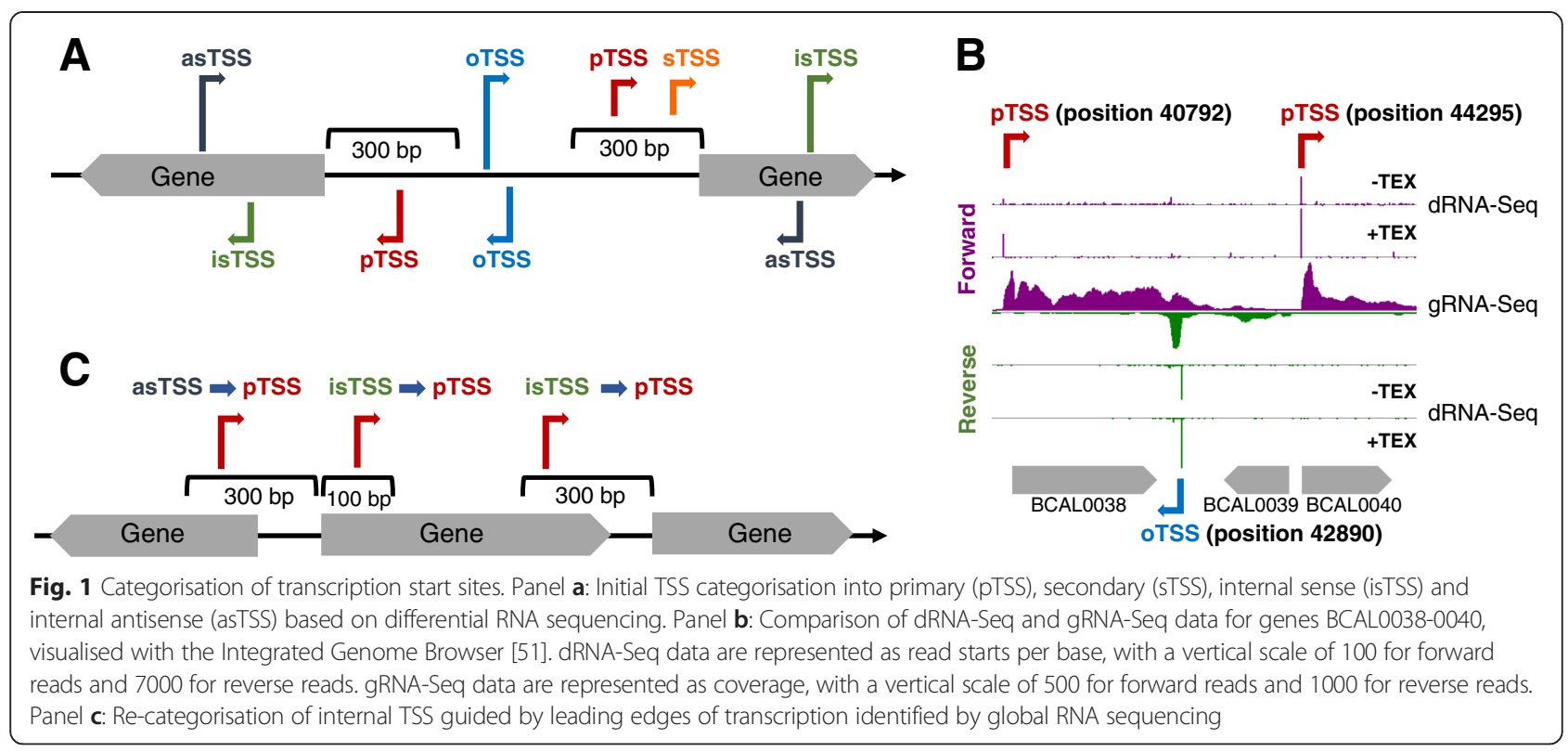

for three genes (BCAL3153, BCAL0301 and BCAL0672) were confirmed by 5 'RACE (Additional file 4: Figure S1, panels A, B and C).

105 pTSS were located internal to an upstream gene (Table 1), in some cases the upstream gene was part of the same operon (Fig. 1). Two internal pTSS located in an upstream same sense gene, one with and one without LE, were analysed by 5'RACE (Additional file 4: Figure S1, panels $E$ and $F$ ). Where a distinct LE was present, the pTSS was unambiguously confirmed, indicating that these adjacent genes do not constitute an operon. Where a LE was not apparent, transcription initiation as well as read-through from further upstream in the operon occurred. This confirms previous observations that transcription can be initiated or modulated at several loci within an operon, resulting in full length transcripts and alternative transcripts [12].

\section{Promoters}

As the present dataset is derived from analysis of only one condition, biofilm growth, promoter search focussed on the core promoter region with its -10 and -35 elements.

Sequences 60 nt upstream of pTSS, excluding TSS located in genomic islands, were submitted to Improbizer [13], a motif finding algorithm that considers location of sequence patterns within the input sequences and favours motifs that occur at the same place. Improbizer found 3 motifs (Additional file 5: Table S4), the first two of which were plausible candidates for a -10 and -35 box based on their sequence and their position relative to TSS. Of the 1733 analysed upstream sequences, more than $95 \%$ possessed a 9 nt long AT-rich motif, on average at position -8 to -16 relative to the TSS (Fig. 2a, Additional file 5: Table S4). The more conserved part of this motif, with consensus sequence TAnAAT, is very similar to the conserved -10 hexamer of $E$. coli with consensus sequence TATAAT, regarding sequence and position relative to TSS [14]. A second, less conserved motif was found in $93 \%$ of submitted sequences, it centred at position -34 (Fig. 2b). Its consensus sequence is TTGCC, making it similar to the conserved -35 box of $E$. coli [14] with consensus sequence TTGACA.

The same 1733 upstream sequences were also analysed with MEME [15], and DMINDA [16], confirming the more conserved first motif, with the same conserved part of the core promoter region containing the -10 consensus sequence TAnAAT and the same positioning relative to TSS (Fig. 2c, d, e). The second less conserved motif could not be confirmed by either MEME or DMINDA, presumably because it is too weak to be detected by algorithms which do not take the position of the motif into account.

The sequences up to $60 \mathrm{nt}$ upstream of internal pTSS, asTSS, isTSS and oTSS were screened for occurrence of the conserved and AT-rich motif (Fig. 2a) to assess whether these TSS were derived from genuine transcription initiation or from sequencing artefacts. We used Motif Finder [13], a program which considers the location of the motif in query sequences when searching for matches. More than $95 \%$ of internal pTSS were associated with a sequence match to the AT-rich motif, on average at the same position as the input motif (Additional file 5: Table S4). Furthermore, $94 \%$ of asTSS, $92 \%$ of oTSS and $81 \%$ of isTSS were associated with a matching motif in the same position.

Overall, the occurrence and position of promoters for transcription initiation further corroborates that most TSS found by dRNA-Seq are bona fide. The lower incidence of promoters for isTSS indicates that isTSS can be also caused by TEX inhibition at strong secondary structures, as has 
A

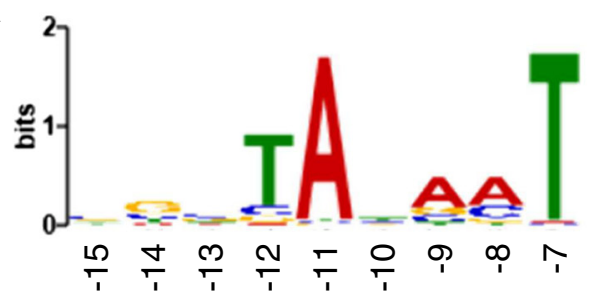

B

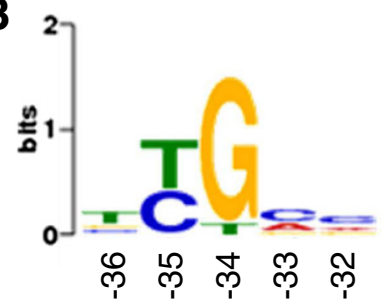

C

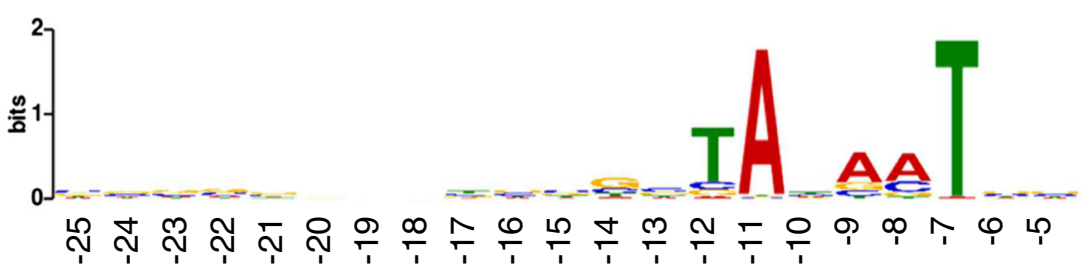

D

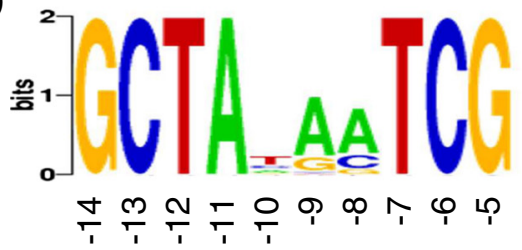

E

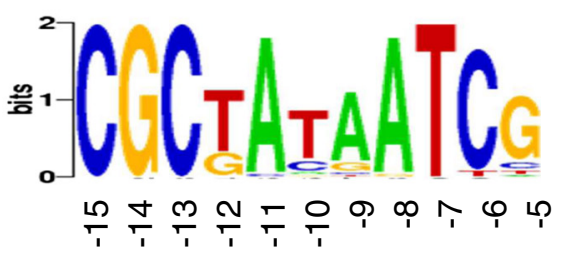

Fig. $\mathbf{2}$ Sequence logos showing conserved motifs upstream of pTSS. Motifs are based on 1733 sequences upstream of primary TSS located in intergenic regions, analysed with Improbizer [13] (panels a and b), MEME [15] (panel c) and DMINDA [16] panel (d and e). X-axis: Average position relative to TSS. Y-axis: Sequence conservation

been observed for Streptomyces coelicolor, another organism with high genomic GC content $[17,18]$.

MEME detected only 5 motifs with an e-value $<0.001$, only one of which, containing the proposed -10-box (Fig. 2c), was plausible as a promoter motif based on its conservation and convergence towards a specific position (Additional file 6: Table S5). DMINDA detected 16 motifs, only two of which, both AT-rich, were converging towards a specific position (Fig. 2d and e, Additional file 7: Table S6). Variations of motifs which could represent different sigma factor binding sites were not found in this analysis. Repeating the analysis with adjusted input paratmeters did not improve results. This is probably due to the relatively large number of input sequences (upstream sequences from all genes expressed under biofilm condition). Analysing subsets of these sequences, generated based on similar expression patterns in a microarray dataset [19] or on related functions, also did not result in plausible specific and conserved sigma factor binding site motifs (data not shown). A reason for this might be the large number of sigma factors encoded in the B. cenocepacia J2315 genome [5]. This bacterium possesses 20 sigma factors the target genes of which have not yet been characterised and which probably have overlapping target gene populations. A more in depth analysis of promoter sequences might therefore require experimental evidence regarding sigma factor target genes, generated e.g. by ChIP sequencing.

\section{Length of 5' UTRs and leaderless transcripts}

The average length of $5^{\prime}$ UTRs is 72 nt (Fig. 3), with a distribution peak between 21 and $30 \mathrm{nt}$; $75 \%$ of $5^{\prime}$ UTRs were between 17 and 126 nt long. This is in good agreement with values for other bacteria such as Salmonella enterica [9], Helicobacter pylori [10] and Streptomyces coelicolor [18].

The length of a 5'UTR can be related to expression regulation of the corresponding gene. Long 5'UTRs may contain riboswitches or provide binding sites for small regulatory RNAs $[20,21]$. Leaderless genes are translated by a different mechanism than genes with a leader sequence, and have been shown to be differentially regulated under stress conditions compared to leader-lead genes [22]. To investigate a possible link between length of 5'UTRs and gene function in B. cenocepacia J2315, we performed functional enrichment analysis on subsets of genes, genes without $(\leq 10 \mathrm{nt}) 5^{\prime} \mathrm{UTR}$ or with a long (>150 nt) 5'UTR.

The pTSS of 72 genes were located exactly at the annotated start codon and the pTSS of a further 42 genes was located $\leq 10$ nt upstream of the annotated start codons. These transcripts were considered to be leaderless. 24 leaderless transcripts were tRNAs, consistent with the length of tRNA leader sequences in B. cenocepacia J2315, which ranges from 5 to 127 nt (see below). Functional enrichment analysis of the remaining leaderless 


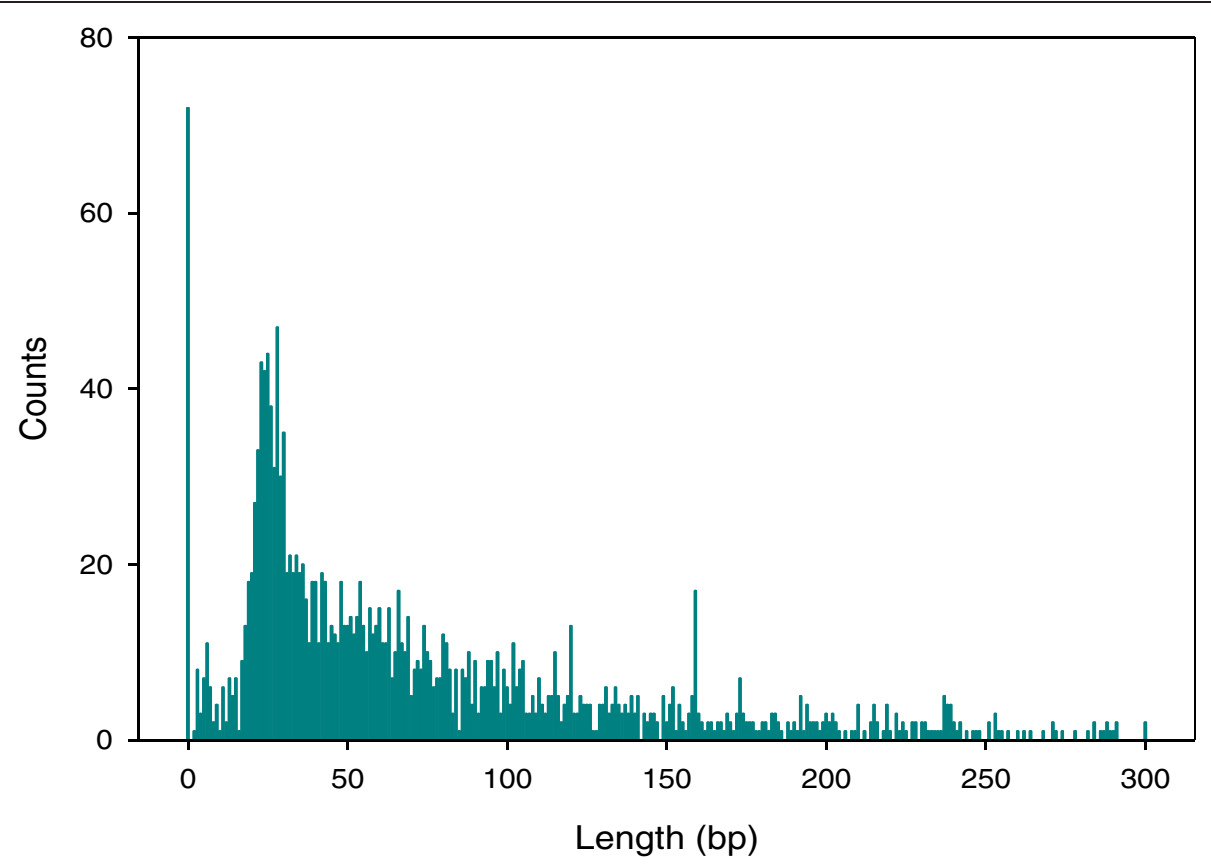

Fig. $35^{\prime}$ UTR length distribution. Data represents $5^{\prime} U$ TRS from primary TSS located in intergenic regions, excluding internal primary TSS

transcripts revealed that, with 28 coding sequences (CDS), transcriptional regulators of various families are particularly over-represented (Additional file 8: Table S7). The TA-rich promoter motif was found directly upstream of genes with leaderless transcripts (Fig. 3), like reported for other bacteria [23], showing that leaderless genes possess a transcription initiation signal instead of a Shine-Dalgarno sequence.

187 CDS featured a long 5'UTR of $>150$ nt. Functional enrichment analysis performed on these genes revealed transcriptional regulators, nucleotide binding and membrane proteins as over-represented (Additional file 8: Table S7). Comparison of the respective sequences with the Rfam database revealed the yet unannotated S-adenosyl-L-homocysteine (SAH) riboswitch [20] in the 5' UTR of an adenosylhomocysteinase (BCAL0145, see below).

\section{Transcription initiation in genomic islands}

The genome of B. cenocepacia J2315 contains 14 genomic islands with a GC-content lower than genome average of $66.9 \%$ or with CDS similar to prophages; these GI encompass $9.3 \%$ of the total genome [5]. Internal TSS appear to occur at a higher density in genomic islands (Fig. 4), in agreement with observations made in E. coli and Salmonella sp. [24]. $18 \%$ of all annotated isTSS and $25 \%$ of asTSS are located in genomic islands, which is higher than expected given the proportion of genomic islands on the genome.

This indicates that, while genes in genomic islands can be transcribed, these loci are not completely compatible with the B. cenocepacia J2315 transcriptional machinery, and transcription initiation often does not result in a functional product [25].

Interestingly, the genomic region encoding exopolysaccharide synthesis genes implicated in capsule formation (BCAL3217-3246) also shows a higher density of internal TSS, thus confirming that this region was acquired by recent gene transfer and constitutes a genomic island [26]. Moreover, most TSS annotated on the plasmid were categorised as internal TSS, indicating that plasmid genes are also not entirely compatible with $B$. cenocepacia transcription mechanisms. We propose that the distribution of internal TSS across the B. cenocepacia genome can be used as a further criterion to identify genomic islands in B. cenocepacia, as genomic islands can display a higher density of internal TSS than genome background.

\section{Re-annotation of start codons and discovery of un-annotated proteins}

Gene calling for genome annotation usually predicts up to $99 \%$ of all protein coding genes correctly, but the rate of gene calling errors can rise to $14 \%$ in the GC-rich Burkholderia sp. genomes [27]. We used TSS mapping to screen for mis-annotated and un-annotated genes in the $B$. cenocepacia 2315 genome.

For 72 genes, 66 CDS and 6 RNAs, TSS mapping predicted the primary TSS internal and downstream of the annotated gene start, suggesting that an incorrect start codon might have been predicted and the gene is shorter than annotated. The internal TSS position for BCAL0063 was confirmed by 5'RACE (Additional file 4: Figure S1, 


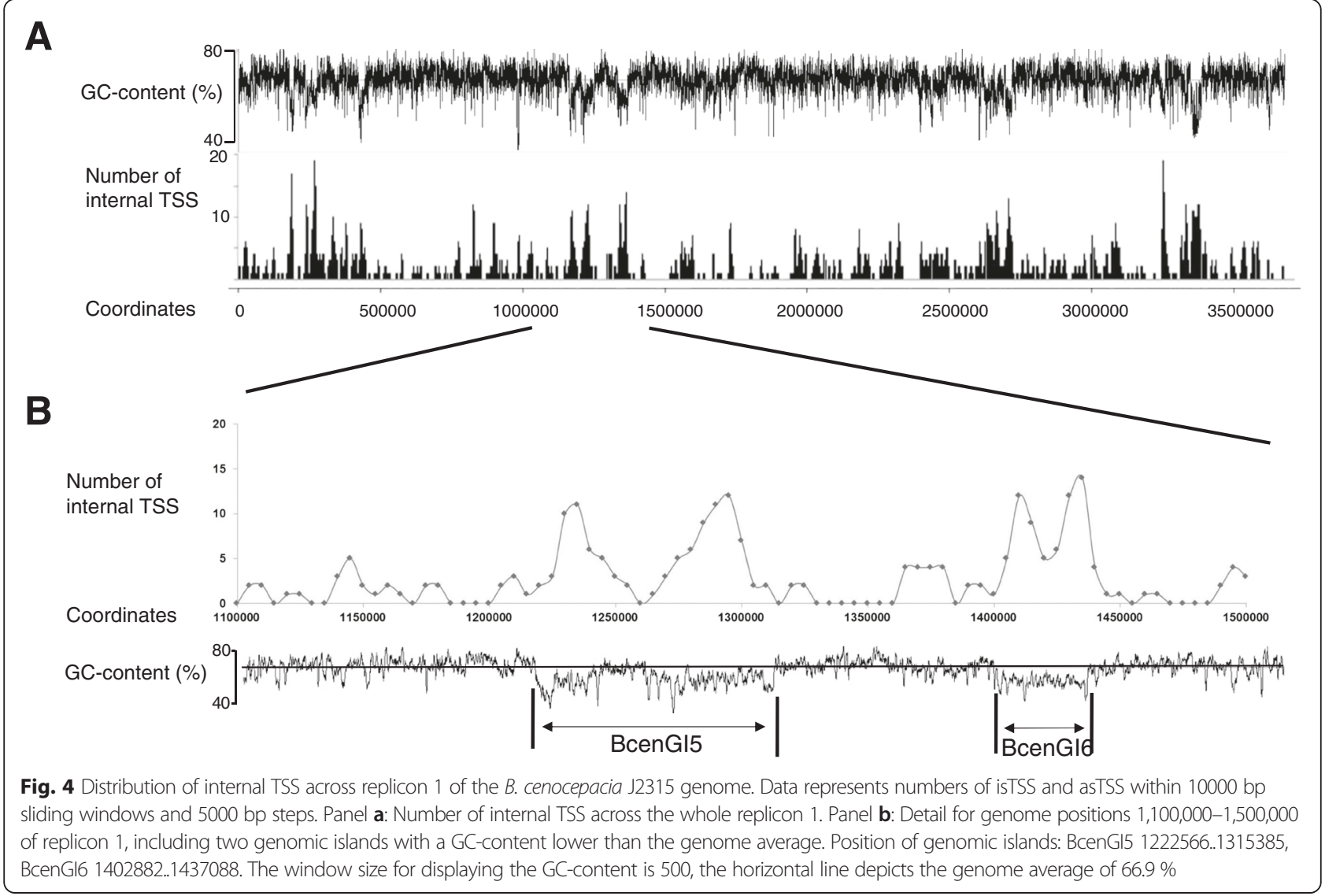

panel D) as an example. All genes with internal and downstream pTSS, as well as leaderless genes, were screened for alternative start codons downstream of their annotated gene start. Unusually long 5'UTRs on the other hand could indicate that the corresponding gene is longer than annotated. 5'UTRs longer than $150 \mathrm{nt}$, as well as orphan TSS consistent with a 5'UTR according to gRNA-Seq data, were screened for open reading frames and upstream alternative start codons. 64 CDS for which an alternative start codon could be predicted, making the gene either longer or shorter, are listed in Table 2.

To search the genome for un-annotated protein-coding genes, all oTSS and asTSS possessing a LE were screened for an open reading frame with an ATG start codon which could produce a protein $\geq 50$ amino acid residues. These amino acid sequences were compared to the NCBI protein sequence database and hits with both $>75 \%$ query coverage and $>40 \%$ amino acid identity were retained (Additional file 9: Table S8). Most hits were annotated as hypothetical or conserved hypothetical proteins with no predicted function. In one case, a type II toxin-antitoxin module was discovered on the opposite strand of a gene currently annotated as BCAL1704, a conserved hypothetical protein. We propose to re-annotate this loci, to BCAL1704A, a ParD-type antitoxin with 81 amino acid residues, and BCAL1704B, a ParE-type toxin with 99 amino acid residues (Fig. 5).

\section{Antisense transcription}

Most reads mapping to annotated genome features map in sense direction ( $>93 \%$ ), only $5-7 \%$ map antisense, based on the gRNA-Seq dataset. Nonetheless, antisense transcription is pervasive in B. cenocepacia J2315, and reads map antisense to nearly all genome features (Additional file 10: Table S9). However, it is safe to assume that not all of these antisense transcripts have a function [28]. Genes with strong sense transcription tend to have a high number of antisense reads, likely a result of technical RNA sequencing artefacts. Moreover, antisense transcription can be a result of read-through from the 3 ' end of a downstream opposite-sense gene or transcription initiation for an upstream opposite-sense gene.

We attempted to annotate antisense transcripts that might genuinely be involved in gene regulation, i.e. transcripts complementary to at least part of a gene and not belonging to any category mentioned above. For this purpose we filtered for genes with a minimum antisense-RPKM of 10 and a ratio of antisense-to-sense RPKM of $>0.1$, leaving $11 \%$ of all genes. 105 of these featured an asTSS, or an oTSS located $\leq 300 \mathrm{bp}$ downstream 
Table 2 Alternative start codons for CDS with internal TSS or long $5^{\prime}$ UTRs $\geq 150 \mathrm{nt}$, as predicted by Prodigal

\begin{tabular}{|c|c|c|c|c|c|c|c|}
\hline TSS position & Strand & Gene & Annotated gene position & $\begin{array}{l}\text { Alternative } \\
\text { gene start }\end{array}$ & $\begin{array}{l}\text { Annotated } \\
\text { start codon }\end{array}$ & $\begin{array}{l}\text { Alternative } \\
\text { start codon }\end{array}$ & $\begin{array}{l}\text { Alternative } \\
\text { gene is }\end{array}$ \\
\hline \multicolumn{8}{|l|}{ Replicon 1} \\
\hline 108753 & + & BCAL0088 & 108758..109138 & 108842 & GTG & ATG & shorter \\
\hline 175808 & + & BCAL0151 & $175965 . .177107$ & 175872 & ATG & ATG & longer \\
\hline 196936 & + & BCAL0175 & 196857..197147 & 196965 & ATG & ATG & shorter \\
\hline 317647 & + & BCAL0289 & $317651 . .322474$ & 317771 & GTG & ATG & shorter \\
\hline 566857 & - & BCAL0515 & Complement (565664..566551) & 566617 & ATG & ATG & longer \\
\hline 700318 & + & BCAL0646 & $700302 . .701309$ & 700341 & GTG & ATG & shorter \\
\hline 787134 & + & BCAL0722 & $787317 . .788582$ & 787293 & GTG & GTG & longer \\
\hline 937522 & + & BCAL0865 & $937677 . .938504$ & 937566 & TTG & ATG & longer \\
\hline 999283 & - & BCAL0916 & Complement (998559..999290) & 999245 & ATG & ATG & shorter \\
\hline 1041114 & - & BCAL0952 & Complement (1040227..1040955) & 1041075 & ATG & ATG & longer \\
\hline 1147555 & + & BCAL1059 & $1147734 . .1148927$ & 1147695 & ATG & ATG & longer \\
\hline 1161150 & - & BCAL1069 & Complement (1159161..1160903) & 1160996 & ATG & ATG & longer \\
\hline 1207883 & - & BCAL1102 & Complement (1207594..1207914) & 1207848 & GTG & ATG & shorter \\
\hline 1390938 & - & BCAL1277 & Complement (1388805..1390997) & 1390868 & ATG & ATG & shorter \\
\hline 1463359 & - & BCAL1335 & Complement (1462753 ..1463376) & 1463334 & ATG & ATG & shorter \\
\hline 1892553 & + & BCAL1715 & $1892545 . .1893150$ & 1892578 & GTG & GTG & shorter \\
\hline 1933621 & + & BCAL1753 & 1933609..1934565 & 1933672 & ATG & ATG & shorter \\
\hline 2041280 & - & BCAL1849 & Complement (2040670..2041326) & 2041221 & ATG & ATG & shorter \\
\hline 2067405 & - & BCAL1871 & Complement (2065350..2067203) & 2067380 & ATG & ATG & longer \\
\hline 2120545 & + & BCAL1921 & 2120767..2121222 & 2120662 & GTG & ATG & longer \\
\hline 2136329 & + & BCAL1937 & $2136797 . .2138446$ & 2136644 & ATG & ATG & longer \\
\hline 2656999 & - & BCAL2401 & Complement (2656576..2657001) & 2656953 & GTG & ATG & shorter \\
\hline 2822661 & - & BCAL2559 & Complement (2822212..2822745) & 2822661 & TTG & ATG & shorter \\
\hline 3011653 & + & BCAL2740 & $3011646 . .3012689$ & 3011679 & ATG & ATG & shorter \\
\hline 3037852 & - & BCAL2766 & Complement (3037324.3037656) & 3037734 & TTG & ATG & longer \\
\hline 3093624 & - & BCAL2818 & Complement (3092132..3093673) & 3093598 & GTG & ATG & shorter \\
\hline 3094006 & + & BCAL2819 & $3093965 . .3095413$ & 3094037 & GTG & ATG & shorter \\
\hline 3122838 & - & BCAL2841 & Complement (3121514..3122860) & 3122710 & GTG & ATG & shorter \\
\hline 3257864 & - & BCAL2974 & Complement (3257551..3257931) & 3257820 & ATG & ATG & shorter \\
\hline 3266225 & + & BCAL2981 & $3266173 . .3268080$ & 3266245 & ATG & ATG & shorter \\
\hline 3461898 & + & BCAL3168 & $3461889 . .3462575$ & 3461937 & ATG & ATG & shorter \\
\hline 3533713 & - & BCAL3229 & Complement (3531274..3533205) & 3533499 & GTG & ATG & longer \\
\hline 3584399 & - & BCAL3275 & Complement (3583374..3584396) & & ATG & TTG & shorter \\
\hline 3619212 & - & BCAL3302 & Complement (3616817..3619048) & 3619129 & GTG & ATG & longer \\
\hline 3666930 & + & BCAL3349 & 3666923..3667387 & 3666965 & ATG & ATG & shorter \\
\hline 3721637 & - & BCAL3395 & Complement (3719319..3721676) & 3721619 & ATG & ATG & shorter \\
\hline \multicolumn{8}{|l|}{ Replicon 2} \\
\hline 14979 & + & BCAM0014 & $15152 . .15757$ & 15110 & ATG & ATG & longer \\
\hline 184425 & - & BCAM0158 & Complement (182630..184141) & 184234 & ATG & ATG & longer \\
\hline 570490 & + & BCAM0516 & $570437 . .570919$ & 570545 & TTG & ATG & shorter \\
\hline 713692 & + & BCAM0645 & $713658 . .715211$ & 713733 & ATG & ATG & shorter \\
\hline 878428 & + & BCAM0795 & $878392 . .878856$ & 878428 & $\pi G$ & ATG & shorter \\
\hline
\end{tabular}


Table 2 Alternative start codons for CDS with internal TSS or long 5' UTRs $\geq 150$ nt, as predicted by Prodigal (Continued)

\begin{tabular}{|c|c|c|c|c|c|c|c|}
\hline 904562 & + & BCAM0820 & $904770 . .905861$ & 904728 & ATG & ATG & longer \\
\hline 1009355 & + & BCAM0918 & $1009947 . .1011812$ & 1009395 & GTG & ATG & longer \\
\hline 1203868 & + & BCAM1112 & 1204104.1206368 & 1204068 & ATG & ATG & longer \\
\hline 1405396 & + & BCAM1280 & 1405387..1406880 & 1405435 & ATG & ATG & shorter \\
\hline 1419414 & + & BCAM1290 & $1419396 . .1420382$ & 1419438 & TTG & ATG & shorter \\
\hline 1967577 & + & BCAM1756 & 1967535..1969922 & 1967598 & GTG & ATG & shorter \\
\hline 2032438 & + & BCAM1814 & $2032438 . .2034045$ & 2032462 & ATG & ATG & shorter \\
\hline 2291422 & - & BCAM2058 & Complement (2290470..2291126) & 2291303 & $\pi G$ & ATG & longer \\
\hline 2291441 & + & BCAM2059 & $2291632 . .2292495$ & 2291587 & ATG & ATG & longer \\
\hline 2300468 & + & BCAM2066 & $2300462 . .2301877$ & 2300489 & ATG & ATG & shorter \\
\hline 2471730 & - & BCAM2210 & Complement (2471467..2471733) & 2471658 & ATG & ATG & shorter \\
\hline 2613643 & + & BCAM2327 & $2613607 . .2614722$ & 2613697 & ATG & ATG & shorter \\
\hline 2703044 & + & BCAM2401 & $2703049 . .2703798$ & 2703109 & ATG & ATG & shorter \\
\hline 2703867 & + & BCAM2402 & $2703876 . .2704169$ & 2703900 & GTG & ATG & shorter \\
\hline 3026129 & + & BCAM2679 & $3026111 . .3026374$ & 3026153 & $\Pi \mathrm{TG}$ & ATG & shorter \\
\hline 3058373 & - & BCAM2703 & Complement (3057460..3058437) & 3058353 & GTG & ATG & shorter \\
\hline 3077665 & - & BCAM2719 & Complement (3077106..3077669) & 3077606 & ATG & ATG & shorter \\
\hline 3133147 & + & BCAM2769 & 3133305.3134042 & 3133281 & GTG & ATG & longer \\
\hline \multicolumn{8}{|c|}{ Replicon 3} \\
\hline 69880 & - & BCAS0060 & Complement (68555..69688) & 69709 & ATG & ATG & longer \\
\hline 80842 & - & BCAS0070 & Complement (79501..80850) & 80742 & TTG & ATG & shorter \\
\hline 769919 & + & BCAS0706 & $770234 . .771562$ & 770120 & TTG & ATG & longer \\
\hline \multicolumn{8}{|l|}{ Plasmid } \\
\hline 2363 & + & pBCA003 & $2363 . .3220$ & 2546 & ATG & ATG & shorter \\
\hline 79981 & - & pBCA080 & Complement (79541..79750) & 79891 & ATG & ATG & longer \\
\hline
\end{tabular}

of the 3'end of a gene, with a LE in gRNA-Seq data (Additional file 11: Table S10). Among the genes with antisense transcription were 12 transposases, 12 transcriptional regulators and two toxin-antitoxin systems, the same categories for which functional antisense transcripts have been found in other bacteria [29]. These antisense RNAs might therefore also have a function in $B$. cenocepacia.

\section{Genes induced under biofilm condition}

To screen the RNA-seq data for genes potentially essential for biofilm growth of B. cenocepacia J2315, we compared RNA-seq data with published microarray datasets obtained from cells grown in a biofilm [30], from planktonic cells harvested in stationary phase, and from cells grown under reduced oxygen levels [19] as well as under various stress conditions $[19,30]$. The aim was to find genes with high expression in biofilms (gRNA-Seq RPKM >100) and induced in biofilms while not induced under any other condition.

Only the first two genes of a multi-subunit $\mathrm{K}^{+}$-transport system ( $k d p A-k d p E$, BCAL2379-2383) met these criteria. These five genes are organised in two operons with two annotated TSS, the first of which is 100-fold stronger expressed than the second (Fig. 6a). The first operon contains the structural $\mathrm{K}^{+}$-transport and ATPase genes, the second contains the two-component regulatory system required for induction of $k d p A B C$. qPCR analysis of $k d p A$ confirmed its induction in biofilms compared to planktonic cultures in logarithmic and stationary phase (Fig. 6b). In $E$. coli, the Kdp-system is an inducible high affinity $\mathrm{K}^{+}$-pump essential for intracellular $\mathrm{K}^{+}$-homoeostasis under salt stress, [31]. The two-component system $k d p D E$ has also been implicated in virulence in various pathogenic bacteria [31]. In Bacillus sp., the Kdpsystem was found to be up-regulated in swarming cells [32] and necessary for biofilm formation [33], and the Kdp-system was up-regulated in Staphylococcus aureus biofilms [34]. These observations indicate that, apart from its role in osmoadaptation, the Kdp-system also plays an important role in biofilm formation, presumably also in $B$. cenocepacia. Work to characterise the relevance of these genes for $B$. cenocepacia biofilm formation and persistence, using deletion and conditional mutants, is ongoing. 


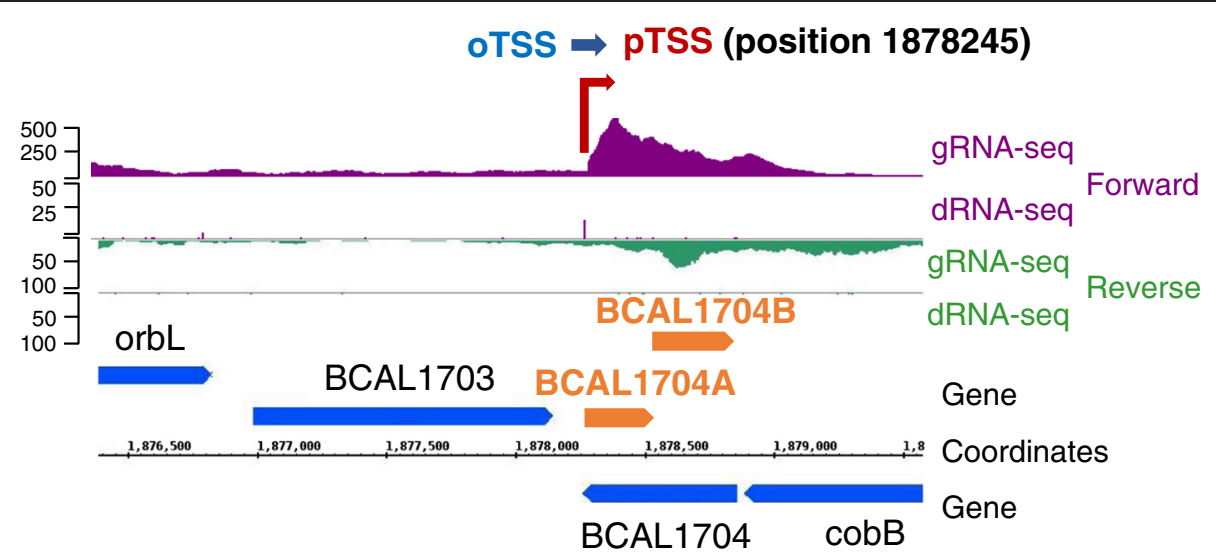

Fig. 5 Re-annotation of a conserved hypothetical protein as a type II toxin-antitoxin system. New annotation: BCAL1704A, ParD, antitoxin component of, and BCAL1704B, ParE, toxin component of a type II toxin-antitoxin system. Position of ParD: 1878263..1878505. Position of ParE: 1878498..1878794. The start of ParE overlaps the 3'end of ParD by $8 \mathrm{nt}$. dRNA-Seq data are represented as read starts per base, gRNA-Seq data are represented as coverage

\section{Transcription of annotated non-coding RNAs}

The published genome annotation [5] includes rRNAs and small non-coding RNAs such as tRNAs, several riboswitches and essential RNAs.

For 47 of the 74 annotated tRNA genes a TSS was found, positioned 5-127 nucleotides upstream of the annotated gene start. Most tRNA transcripts are, as expected, processed at position +1 of the annotated gene, marked by a local maximum in read starts in the -TEX library and depletion in the + TEX library.

Exceptions are the tRNAs for histidine and selenocysteine, which are processed at position -1 and -10 , respectively. 16S rRNA genes have a TSS at position -237 , the co-transcribed 23S and 5S rRNA are processed at +3 and +2 , respectively. TSS for annotated riboswitches are positioned at annotated gene start (thiamine pyrophosphate) and 5 nt (glycine) or 8-14 nt upstream (cobalamin).

Of the annotated essential RNAs, transfer messenger RNA has an 11 bp 5'-leader element. RNase P appears longer than annotated, its TSS is located at position -51 and the transcript shows no obvious processing site. The signal recognition particle appears shorter than annotated, with a TSS at position +2 .

Overall, dRNA-Seq data are in good agreement with annotated non-coding RNAs, showing that small RNAs are detected with our experimental approach.

\section{Candidate regulatory small RNAs}

Apart from annotated tRNAs, riboswitches and conserved essential RNAs, bacterial genomes also contain other small non-coding RNAs that are involved in post transcriptional gene expression regulation [21, 35]. For a preliminary screening of TSS for highly expressed novel small RNAs, sequences following oTSS, isTSS and asTSS, as well as 5' UTRs longer than the average of $72 \mathrm{nt}$, were compared to the Rfam database.
In this manner nine small RNAs were found in the $B$. cenocepacia J2315 genome (Table 3). One of these constitutes the 6S RNA, which was already predicted and its expression confirmed by other studies on B. cenocepacia $[30,36]$. Two small RNAs are phage-related regulatory RNAs located on genomic island BcenGI9. Two Rfam hits constitute conserved regulatory motifs: the SAH riboswitch located in the 5'UTRs of BCAL0145, an adenosylhomocysteinase, and the Burkholderiales-specific sucA RNA motif located in the 5'UTR of BCAL1515, sucA, an enzyme of the citric acid cycle. The sucA RNA motif probably constitutes a riboswitch [37]. The remaining four novel small RNAs all are from the same family, "toxic small RNAs", which were found to be toxic if introduced into $E$. coli on a cloning vector [38]. Expression of these toxic small RNAs has been confirmed by Northern blotting in four strains of B. cenocepacia, including strain J2315 [38]. However, their function in B. cenocepacia is unknown.

The sequence of small RNAs is generally only conserved between closely related bacterial species and can vary dramatically in primary sequence and secondary structure between bacterial genera [39]. On the other hand, most small RNAs in the Rfam database are derived from wellstudied species such as E. coli, which are not closely related to Burkholderia. Since the Rfam algorithm first performs a BLAST search, functional homologues from distantly related species are unlikely to produce a hit and novel small RNAs specific for B. cenocepacia are likely to be overlooked by this approach.

To identify putative regulatory small non-coding RNAs not yet represented in the Rfam database, we compared sequences derived from oTSS to non-coding RNAs experimentally confirmed in B. cenocepacia strains in other studies using RNA-sequencing and Northern blotting [36], co-purification with Hfq-protein [40] or microarrays $[30,41-43]$, if they showed the following properties: 


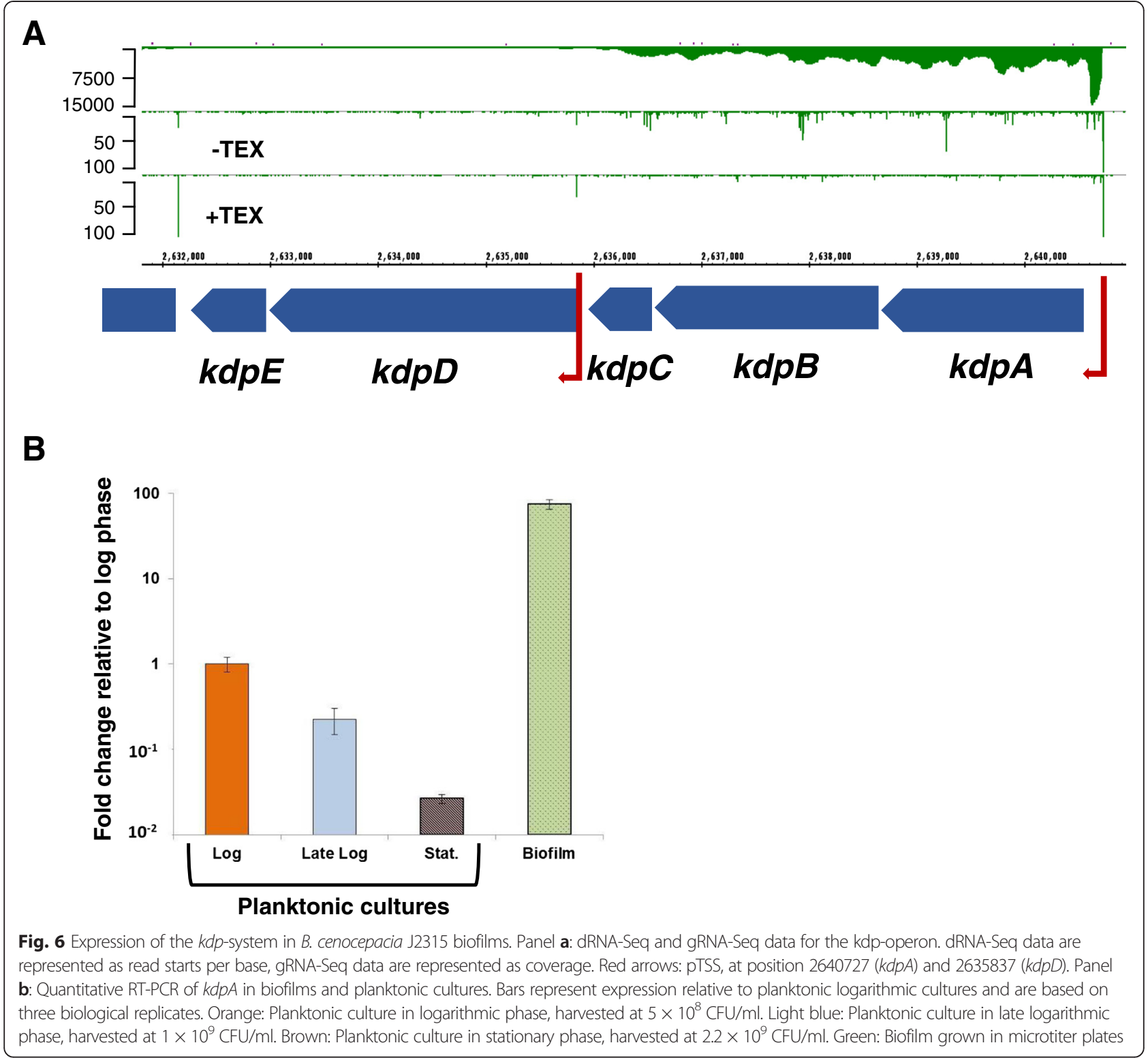

strong transcription initiation with a coverage $>300$ reads in dRNA-Seq data, a defined 3' end in dRNA-Seq data or a transcript appearing short $(<500 \mathrm{nt})$, truncated or missing in gRNA-Seq data.

Homologues of six short transcripts with strong transcription initiation from oTSS were also expressed in $B$. cenocepacia strains AU1054 and HI2424 under conditions mimicking the human lung and the soil environment (Table 4) [36]. One of these was confirmed to be a small RNA by Northern blotting in the same study. One short transcript was present in the RNA fraction copurified with the Hfq-protein of B. cenocepacia J2315 [40]. $\mathrm{Hfq}$ is an RNA chaperone which mediates base pairing of small regulatory RNAs with their target mRNA [44] and B. cenocepacia J2315 harbours the Hfq gene as two non- identical homologues [5], making this non-coding RNA a plausible candidate for a regulatory small RNA. These findings show that with the approach used in this study we could identify transcripts which could encode noncoding regulatory small RNAs. Their strong expression in biofilms suggests that these small RNAs might have a role in adaptation of B. cenocepacia to biofilm conditions.

A detailed analysis of putative novel small non-coding RNAs expressed in B. cenocepacia J2315 biofilms and their involvement in biofilm formation is ongoing.

\section{Conclusions}

This study is the first genome-wide analysis of TSS in $B$. cenocepacia. Through differential RNA-Sequencing, bioinformatics methods and 5'RACE we annotated the 
Table 3 Novel non-coding small RNAs in B. cenocepacia J2315 with hits in Rfam database

\begin{tabular}{|c|c|c|c|c|c|c|c|c|c|}
\hline \multirow{2}{*}{$\begin{array}{l}\text { Preliminary } \\
\text { name } \\
\text { ncSO3 }\end{array}$} & \multirow{2}{*}{$\begin{array}{l}\text { Strand } \\
+\end{array}$} & \multirow{2}{*}{$\begin{array}{l}\text { Length }(\mathrm{nt})^{\mathrm{b}} \\
58\end{array}$} & \multirow{2}{*}{$\begin{array}{l}\begin{array}{l}\text { Terminator } \\
\text { sequence }\end{array} \\
\text { yes }\end{array}$} & \multicolumn{2}{|c|}{ Adjacent genes } & \multirow{2}{*}{$\begin{array}{l}\text { Relative } \\
\text { orientation }\end{array}$} & \multirow{2}{*}{$\begin{array}{l}\text { Genome position } \\
221314.221371\end{array}$} & \multirow{2}{*}{$\begin{array}{l}\text { Rfam ID } \\
\text { RF02278 }\end{array}$} & \multirow{2}{*}{$\begin{array}{l}\text { Name } \\
\text { Toxic small RNA }\end{array}$} \\
\hline & & & & BCAL0197 & BCAL0198 & & & & \\
\hline ncS05 & - & 67 & yes & BCAL0436 & BCAL0437 & $\leftarrow \leftarrow \rightarrow$ & Complement (479440..479506) & RF02278 & Toxic small RNA \\
\hline ncS17 & + & 200 & yes & BCAL2667 & BCAL2668 & $\rightarrow \rightarrow \rightarrow$ & 2935785.2935984 & RF00013 & $6 \mathrm{~S}$ RNA \\
\hline ncS23 & + & 81 & yes & BCAL2965 & BCAL2965a & $\rightarrow \rightarrow \rightarrow$ & 3246834.3246914 & RF01394 & isrk \\
\hline ncS24 & + & 90 & no & BCAL2965 & BCAL2965a & $\rightarrow \rightarrow \rightarrow$ & $3246937 . .3247026$ & RF01695 & $C 4^{\mathrm{a}}$ \\
\hline ncS27 & - & 92 & yes & BCAL3348a & BCAL3349 & $\rightarrow \leftarrow \rightarrow$ & Complement (3666557..3666648) & RF02278 & Toxic small RNA \\
\hline ncS62 & + & 57 & yes & BCAM1871 & BCAM1872 & $\rightarrow \rightarrow \leftarrow$ & $2089713 . .2089769$ & RF02278 & Toxic small RNA \\
\hline ncR1 & + & 119 & no & BCAL0144 & BCAL0145 & $\rightarrow \rightarrow \rightarrow$ & $168973 . .169091$ & RF01057 & SAH riboswitch \\
\hline$n c R 2$ & + & 118 & no & BCAL1514 & BCAL1515 & $\rightarrow \rightarrow \rightarrow$ & $1676458 . .1676575$ & RF01070 & SucA RNA motif \\
\hline
\end{tabular}

${ }^{\mathrm{a}} \mathrm{C} 4$ forms one transcriptional unit with isrk

bength of non-coding RNAs is inferred from dRNA-Seq data and terminator structures (when present) and is not yet experimentally confirmed

primary TSS for 2089 genes expressed in biofilms, defined alternative start codons for 64 genes, identified novel protein sequences and characterised antisense transcription. 15 non-coding RNAs highly expressed in biofilms and a potassium uptake system strongly induced under biofilm conditions were identified that could be involved in biofilm formation and survival. Comparison of dRNA-Seq data with gRNA-Seq data proved to be invaluable for TSS categorisation and interpretation.

The data presented in this study will provide the starting point for evaluation of the regulatory processes involved in B. cenocepacia biofilm formation and could reveal novel targets for antibiotic therapy.

\section{Methods}

\section{Bacterial strain and culture conditions}

B. cenocepacia strain J2315 (LMG 16656) was grown in Luria-Bertani broth (LBB, Oxoid, Hampshire, UK) at $37^{\circ} \mathrm{C}$. Biofilms were grown in 96-well microtiter plates and cells were harvested as described previously [45]. Planktonic cultures were grown in $250 \mathrm{ml}$ glass flasks, incubated at $37{ }^{\circ} \mathrm{C}$ in a shaking incubator at $150 \mathrm{rpm}$ and harvested as described previously [46].

\section{RNA extraction and sequencing}

For differential RNA-sequencing (dRNA-Seq, [6]), total RNA was extracted from cell pellets of two biological biofilm replicates using the RiboPure Bacteria kit (Life Technologies, Renfrewshire, UK). RNA samples were then split and one aliquot treated with Terminator ${ }^{\mathrm{Tm}} 5^{\prime}$ monophosphate-dependent exonuclease (TEX). A separate library was constructed from each aliquot, TEX-treated and untreated. rRNA depletion and RNA fragmentation steps were omitted. 5 'triphosphates were removed using tobacco acid pyrophosphatase and an RNA adapter was ligated to the 5'-monophosphate of the RNA. RNA was then polyadenylated and first-strand cDNA synthesis performed using an oligo(dT)-adapter primer. The resulting cDNA was PCR-amplified to about $20-30 \mathrm{ng} / \mu \mathrm{l}$. A libraryspecific barcode for multiplex sequencing was part of a $3^{\prime}$ sequencing adapter. The following adapter sequences flank the cDNA inserts: TrueSeq Sense primer 5'AATGATAC

Table 4 Putative novel non-coding RNAs expressed in B. cenocepacia and experimentally confirmed by previous studies

\begin{tabular}{|c|c|c|c|c|c|c|c|c|c|}
\hline \multirow{2}{*}{$\begin{array}{l}\text { Preliminary } \\
\text { name } \\
\text { ncSO4 }\end{array}$} & \multirow{2}{*}{$\begin{array}{l}\text { Strand } \\
+\end{array}$} & \multirow{2}{*}{$\begin{array}{l}\text { Length }(\mathrm{nt})^{\mathrm{b}} \\
105\end{array}$} & \multirow{2}{*}{$\begin{array}{l}\text { Terminator } \\
\text { sequence }\end{array}$} & \multicolumn{2}{|c|}{ Adjacent genes } & \multirow{2}{*}{$\begin{array}{l}\text { Relative } \\
\text { orientation } \\
\leftarrow \rightarrow \leftarrow\end{array}$} & \multirow{2}{*}{$\begin{array}{l}\text { Genome position } \\
292949 . .293053\end{array}$} & \multirow{2}{*}{$\begin{array}{l}\text { Replicon } \\
1\end{array}$} & \multirow{2}{*}{$\begin{array}{l}\text { Type of } \\
\text { experiment } \\
\text { Co-purification } \\
\text { with Hfq [40] }\end{array}$} \\
\hline & & & & BCAL0264 & BCAL0265 & & & & \\
\hline ncS06 & + & 117 & no & BCAL0549 & BCAL0550 & $\rightarrow \rightarrow \leftarrow$ & $603652 . .603828$ & 1 & $\begin{array}{l}\text { RNA-Seq and } \\
\text { Northern blot [36] }\end{array}$ \\
\hline ncS11 & - & 208 & yes & BCAL2293 & BCAL2294 & $\rightarrow \leftarrow \leftarrow$ & Complement (2545296..2545503) & 1 & RNA-Seq [36] \\
\hline ncS18 & + & 178 & no & BCAL2713 & BCAL2714 & $\rightarrow \rightarrow \rightarrow$ & $2979006 . .2979183$ & 1 & RNA-Seq [36] \\
\hline $\mathrm{ncS} 21^{\mathrm{a}}$ & + & 361 & yes & BCAL2737 & BCAL2738 & $\rightarrow \rightarrow \rightarrow$ & $3008232 . .3008591$ & 1 & RNA-Seq [36] \\
\hline ncS33 & + & 93 & no & BCAM1725 & BCAM1726 & $\rightarrow \rightarrow \rightarrow$ & 1926664..1926756 & 2 & RNA-Seq [36] \\
\hline ncS36 & + & 60 & no & BCAM2207 & BCAM2208 & $\leftarrow \rightarrow \rightarrow$ & $2468880 . .2468939$ & 2 & RNA-Seq [36] \\
\hline
\end{tabular}

${ }^{a}$ ncS21 is associated with an open reading frame and potentially constitutes a protein, see Additional file 6: Table S5

bength of non-coding RNAs is inferred from dRNA-Seq data and terminator structures (when present) and is not yet experimentally confirmed 
GGCGACCACCGAGATCTACACTCTTTCCCTACACG ACGCTCTTCCGATCT-3' and TrueSeq Antisense NNNNNN primer $(\mathrm{NNNNNN}=6 \mathrm{n}$ barcode for multiplexing) 5'-CAAGCAGAAGACGGCATACGAGATNNNNNN-GTGACTGGAGTTCAGACGTGTGCTCT TCCGATC(dT25)-3'. The resulting cDNA libraries were sequenced using a HiSeq 2000 machine (Illumina) in single-read mode and running 100 cycles.

For global transcriptome sequencing (gRNA-Seq), RNA of three biological biofilm replicates was extracted as described previously [45]. Total RNA was depleted of ribosomal RNA using the Ribo-zero Magnetic Gram-Negative Bacteria kit (Epicentre, Madison, WI, USA) and used for Illumina paired-end sequencing generating $100 \mathrm{bp}$ reads [45].

\section{Mapping of RNA seq reads}

In order to assure a high sequence quality, the Illumina reads in FASTQ format were trimmed with a cut-off phred score of 20 by the program fastq_quality_trimmer from FASTX toolkit version 0.0.13 (http://hannonlab.cshl.edu/ fastx_toolkit/). The following steps were performed using the subcommand "create", "align" and "coverage" of the tool READemption [47] version 0.3.5. The poly(A)-tail sequences were removed and a size filtering step was applied in which sequences shorter than $12 \mathrm{nt}$ were eliminated. The collections of remaining reads were mapped to the reference genome sequences (accessions AM747720, AM747721, AM747722, and AM747723) using segemehl software version 0.2.0 [48]. Coverage plots in wiggle format representing the number of aligned reads per nucleotide were generated based on the aligned reads just considering the first base of each read.

\section{TSS annotation and classification}

Mapping output from dRNA-Seq was split by replicon and coverted to SAM format (using samtools 1.2). Those SAM files were used as input for TSSAR, a tool for automated de novo TSS annotation [49], to map all loci with coverage maxima which are enriched in the TEXtreated library. Default parameters ( $\mathrm{p}$-value threshold 0.001 , noise threshold 2, merge range 5) were used, the two biological replicates were pooled and TSS within $5 \mathrm{nt}$ of each other were clustered into one. Genome regions with read start distributions that do not conform to a Poisson distribution are omitted from TSSAR analysis [49]. Such regions were then manually annotated by scanning the respective wiggle files for nucleotides with an abrupt increase in coverage; except regions internal to rRNA genes where the abundance of mapped reads was too high to allow robust TSS annotation. A noise filter with a minimum coverage of 10 read starts was applied, based on the normalised number of read starts per base, to ensure reported TSS are robust. TSS were then classified according to their genomic context. All TSS positions were assigned relative to the start of the associated annotated gene, with the first base of the gene being position +1 , upstream positions start with -1 .

We used the B. cenocepacia strain J2315 annotation deposited in the EMBL database under accession numbers AM747720, AM747721, AM747722, and AM747723 [5] for assigning and classifying TSS. This newer annotation includes 21 non-coding RNAs other than rRNAs and tRNAs: riboswitches for e.g. thiamine and cobalamin and the essential RNAs tmRNA, ribonuclease $\mathrm{P}$ and bacterial signal recognition particle. In contrast to this, the older annotation deposited at NCBI under accession numbers NC_011000, NC_011001, NC_011002 and NC_011003 contains only rRNAs and tRNAs and lacks all genes annotated as pseudogenes in the newer annotation.

\section{Rapid amplification of CDNA ends (RACE)}

To confirm TSS, the $5^{\prime}$ end of selected transcripts was determined by RACE. For 5'RACE, RNA was transcribed with gene specific primers and a homopolymeric tail added to the $3^{\prime}$ end of the resulting cDNA. The tailed cDNA was then amplified with nested gene specific primers and a primer complementary to the homopolymer tail. We used the 5' RACE System for Rapid Amplification of cDNA Ends (Life Technologies, Paisley, UK) with the following changes to standard protocol: the reverse transcriptase provided with the kit was replaced by ThermoScript reverse transcriptase (Life Technologies, Paisley, UK), temperature for first strand synthesis was elevated to $60{ }^{\circ} \mathrm{C}$ and the additional protocol for transcripts with high $\mathrm{GC}$-content was followed. The resulting amplicons were cloned into E. coli using the pGEM ${ }^{\circ} \mathrm{T}$ Vector system (Promega, WI, USA) and JM109 high-efficiency competent cells (Promega, WI, USA). Vector inserts amplified from clones were analysed by Sanger-sequencing. RACE primer sequences are available as supplementary data (Additional file 12: Table S11).

\section{Quantitative RT-PCR analysis}

For quantitative RT-PCR (qPCR), planktonic and biofilm cultures were grown and harvested as described previously $[45,46]$. RNA extraction from cell pellets was performed with a modified protocol, using the RiboPure Bacteria kit (Life Technologies, Renfrewshire, UK) with the following changes to manufacturer's instructions: before transferring the RNA to the filter cartridge, 1.25 instead of 0.5 volumes of ethanol were added to retain a higher proportion of small RNAs. Before DNase treatment, RNA was denatured by heating to $65^{\circ} \mathrm{C}$ for $5 \mathrm{~min}$ and DNase incubation time was increased from $30 \mathrm{~min}$ to $60 \mathrm{~min}$. The RNA extract was then DNase-digested (NEB, Ipswich, MA, USA) for a second time for $60 \mathrm{~min}$ and extracted with phenol-chloroform (Roti-Aqua-P/C/I 
for RNA extraction, Carl Roth, Karlsruhe, Germany). Extracted RNA was precipitated with 2.5 volumes ethanolsodium acetate mix (ethanol : $3 \mathrm{M} \mathrm{Na}$-acetate 30:1, $\mathrm{pH}$ 6.5) over night at $-20{ }^{\circ} \mathrm{C}$, centrifuged and washed with $70 \%$ ethanol. The RNA pellet was air dried and re-dissolved in water.

cDNA generation and quantitative RT-PCR was performed as described previously [50] using eight control genes with minimal expression changes across all tested conditions for data normalisation. All eight control genes were used for normalisation in every condition. Primer sequences of target and control genes are shown in Additional file 10: Table S9.

\section{Further bioinformatical analysis}

dRNA-Seq and gRNA-Seq data were visualised with the Integrated Genome Browser version 8.3.1. [51] for manual comparison. Novel proteins were searched for by comparing sequences to the NCBI non-redundant protein sequence database using pBLAST [52], novel noncoding small RNAs were searched for by comparing DNA sequences to the Rfam database [39]. The TransTerm algorithm [53] was used to screen for Rho-independent transcriptional terminator structures. Functional enrichment analysis was performed with the DAVID web tool [54], using a custom background gene list consisting of all genes with an assigned pTSS. Alternative start codons were predicted using Prodigal [55]. DNA sequence motifs upstream of pTSS were identified with Improbizer [13] using default parameters and motifs were then searched for in sequences upstream TSS belonging to other categories with Motif Matcher [13]; both programs consider location of the motif. Improbizer and Motif Matcher are available as web tools at https://users.soe.ucsc.edu/ kent/improbizer/ index.html. Sequences upstream of pTSS were also submitted to MEME [15] and DMINDA [16] for comparison and cross-validation. Input parameters were default, except for minmum and maximum motif length in MEME which were 8 and 50 , respectively.

\section{Supporting data and software}

The dRNA-Seq raw sequencing data was submitted to ArrayExpress under accession number E-MTAB-3381. The gRNA-Seq raw reads are available in ArrayExpress under accession number E-MTAB-2079 [45]. A script that performs the READemption and TSSAR based analysis can be retrieved from https://zenodo.org/record/ 17358 (DOI: 10.5281/zenodo.17358).

Plots for read starts per nucleotide from dRNA-Seq and for coverage from gRNA-Seq as well as data for TSS, candidate regulatory RNAs and alternative annotations are available on the Burkholderia genome database [56] beta site (www.burkholderia.com).

\section{Additional files}

Additional file 1: Table S1. Number of mapped reads per RNA sample. (XLSX $11 \mathrm{~kb}$ )

Additional file 2: Table S2. Transcription start sites identified by differential RNA sequencing and automated annotation. (XLSX 489 kb)

Additional file 3: Table S3. Annotated and categorised TSS in the $B$. cenocepacia 2315 genome. (XLSX 353 kb)

Additional file 4: Figure S1. Transcription start sites confirmed by 5' RACE. dRNA-Seq data are represented as number of read starts per base and gRNA-Seq data as read coverage, both are visualised using the Integrated Genome Browser [51]. dRNA-Seq data are represented as read starts per base, gRNA-Seq data are represented as coverage. Blue arrows depict representative sequences derived from 5'RACE analysis. Panel A: pTSS of BCAL3153, confirmed by 2 out of 8 RACE sequences, 6 sequences were shorter than the putative $5^{\prime} \cup T R$. Panel B: pTSS of BCAL0672, confirmed by 5 out of 12 RACE sequences, 7 sequences were shorter than the putative $5^{\prime} U T R$. The isTSS internal to BCAL0672 was not confirmed by 5'RACE, transcripts originating from this locus are probably truncated. Panel C: pTSS of BCAL0301 confirmed by 2 out of 7 RACE sequences, 5 sequences were shorter than the putative $5^{\prime} U T R$. The isTSS located internal to BCAL0300 could not be confirmed, transcripts originating from this locus are probably truncated. Panel D: Internal pTSS of BCAL0063. 4 out of 5 RACE sequences confirmed the internal pTSS, one read was shorter. Panel E: isTSS within BCAL3201: 3 out of 9 RACE sequences confirmed the isTSS, 3 were shorter and 3 longer than the transcript originating at this TSS. Sequences were therefore derived from transcripts originating from this TSS as well as from TSS further upstream in the operon. Panel F: pTSS for BCAL3391, internal to BCAL3390: confirmed by 6 out of 6 RACE sequences. (PDF $114 \mathrm{~kb}$ )

Additional file 5: Table S4. Promoter motifs in sequences upstream of TSS based on Improbizer [13] analysis. The last nt of all sequences is position +1 of the transcript, the TSS. Motifs with scores $>2$ were regarded as positive matches. Motif sequences are indicated in capital letters. (XLSX 436 kb)

Additional file 6: Table S5. Promoter motifs based on MEME [15] analysis. (XLSX $124 \mathrm{~kb}$ )

Additional file 7: Table S6. Promoter motifs based on DMINDA [16] analysis. Motif sequences are shown in context with the expression pattern of the respective genes, based on microarray data [19]. (XLSX 319 kb)

Additional file 8: Table S7. Genes belonging to enriched functionally related gene groups among leaderless transcripts and among transcripts with a 5'UTR longer than $150 \mathrm{nt}$. (XLSX $12 \mathrm{~kb}$ )

Additional file 9: Table S8. Amino acid sequences associated with orphan TSS or internal antisense TSS and with homologues in the non-redundant protein sequence database. (XLSX 18 kb)

Additional file 10: Table S9. Number of mapped reads and RPKM values sense and antisense for all genes of the B. cenocepacia J2315 genome. (XLSX $2108 \mathrm{~kb}$ )

Additional file 11: Table S10. Genes with antisense transcripts. (XLSX $20 \mathrm{~kb}$ )

Additional file 12: Table S11. Oligonucleotides used in this study. (DOCX $17 \mathrm{~kb})$

\section{Abbreviations}

Bcc: Burkholderia cepacia complex; BcenGl: Burkholderia cenocepacia genomic island; bp: Base pairs; cDNA: Complementary DNA; CDS: Coding sequence; CFU: Colony forming units; dRNA-Seq: Differential RNA sequencing; gRNASeq: Global RNA sequencing; Hfq: Host factor q; kdp: Potassium transport pump; LBB: Luria-Bertani broth; LE: Leading edge of transcription; Mb: Mega base pairs; nt: Nucleotide; NTP: nucleoside triphosphate; TEX: Terminator exonuclease; tRNA: Transfer RNA; rRNA: Ribosomal RNA; RACE: Rapid amplification of cDNA ends; RPKM: Reads per kilo base per million mapped reads; SAH: S-adenosyl-L-homocysteine; UTR: Untranslated region; TSS: Transcription start site (pTSS: Primary TSS, asTSS: Internal antisense TSS, isTSS: Internal sense TSS, oTSS: Orphan TSS, sTSS: Secondary TSS). 


\section{Competing interests}

The authors declare no competing financial and non-financial interests.

\section{Authors' contributions}

AS analysed the RNA-seq data, performed quantitative RT-PCR and RACE experiments and wrote the manuscript. HvA extracted all total RNA for sequencing and submitted gRNA-Seq data to ArrayExpress. FvN and DD performed global RNA sequencing and JV performed differential RNA sequencing. KF provided initial dRNA-Seq data analysis and scripts for transformation of dRNA-Seq data. TC conceived the study, acquired funding and provided research facilities. All authors read and approved the final version of the manuscript.

\section{Authors' information}

AS, HvA, TC: Laboratory of Pharmaceutical Microbiology, Ghent University, Ghent, Belgium.

KF: Core Unit Systems Medicine, University of Würzburg, Würzburg, Germany FvN, DD: Laboratory of Pharmaceutical Biotechnology, Ghent University, Ghent, Belgium.

JV: Institute for Molecular Infection Biology, University of Würzburg, Würzburg, Germany.

\section{Acknowledgements}

This study was funded by the Belgian Science Policy Office within their Interuniversity Attraction Poles (IAP) program, (Phase VII/2012-2017, Project P7/28). The authors wish to thank Bert Remmerie for help with TSS categorisation.

\section{Author details}

${ }^{1}$ Laboratory of Pharmaceutical Microbiology, Ghent University, Ottergemsesteenweg 460, 9000 Ghent, Belgium. ' Laboratory of Pharmaceutical Biotechnology, Ghent University, Ghent, Belgium. ${ }^{3}$ Core Unit Systems Medicine, University of Würzburg, Würzburg, Germany. ${ }^{4}$ Institute for Molecular Infection Biology, University of Würzburg, Würzburg, Germany.

\section{Received: 6 May 2015 Accepted: 6 October 2015}

\section{Published online: 13 October 2015}

\section{References}

1. Peeters C, Zlosnik JEA, Spilker T, Hird TJ, LiPuma JJ, Vandamme P. Burkholderia pseudomultivorans sp. nov., a novel Burkholderia cepacia complex species from human respiratory samples and the rhizosphere. Syst Appl Microbiol. 2013;36(7):483-9.

2. Mahenthiralingam E, Urban TA, Goldberg JB. The multifarious, multireplicon Burkholderia cepacia complex. Nat Rev Microbiol. 2005;3(2):144-56.

3. LiPuma JJ. Update on the Burkholderia cepacia complex. Curr Opin Pulm Med. 2005;11(6):528-33.

4. Coenye T. Social interactions in the Burkholderia cepacia complex: biofilms and quorum sensing. Future Microbiol. 2010;5(7):1087-99.

5. Holden MTG, Seth-Smith HMB, Crossman LC, Sebaihia M, Bentley SD, CerdeñoTárraga AM, et al. The genome of Burkholderia cenocepacia J2315, an epidemic pathogen of cystic fibrosis patients. J Bacteriol. 2009;91(1):261-77.

6. Sharma CM, Vogel J. Differential RNA-seq: the approach behind and the biological insight gained. Curr Opin Microbiol. 2014;19:97-105.

7. Lin YF, A D, Guan S, Mamanova L, McDowall KJ. A combination of improved differential and global RNA-seq reveals pervasive transcription initiation and events in all stages of the life-cycle of functional RNAs in Propionibacterium acnes, a major contributor to wide-spread human disease. BMC Genomics. 2013;14(1):620.

8. Goldman SR, Sharp JS, Vvedenskaya IO, Livny J, Dove SL, Nickels BE. NanoRNAs prime transcription initiation in vivo. Mol Cell. 2011;42(6):817-25.

9. Kröger C, Dillon SC, Cameron AD, Papenfort K, Sivasankaran SK, Hokamp K, et al. The transcriptional landscape and small RNAs of Salmonella enterica serovar Typhimurium. Proc Natl Acad Sci U S A. 2012;109(20):E1277-1286.

10. Sharma CM, Hoffmann S, Darfeuille F, Reignier J, Findeiß S, Sittka A, et al. The primary transcriptome of the major human pathogen Helicobacter pylori. Nature. 2010;464(7286):250-5.

11. Juhas $M$, Stark $M$, von Mering C, Lumjiaktase P, Crook DW, Valvano MA, et al. High confidence prediction of essential genes in Burkholderia cenocepacia. PLOS ONE. 2012;7(6):e40064.

12. Güell M, Yus E, Lluch-Senar M, Serrano L. Bacterial transcriptomics: what is beyond the RNA horiz-ome? Nat Rev Microbiol. 2011;9(9):658-69.
13. Ao W, Gaudet J, Kent WJ, Muttumu S, Mango SE. Environmentally induced foregut remodeling by PHA-4/FoxA and DAF-12/NHR. Science. 2004;305(5691):1743-6.

14. Hawley DK, McClure WR. Compilation and analysis of Escherichia coli promoter DNA sequences. Nucleic Acids Res. 1983;11(8):2237-55.

15. Bailey TL, Johnson J, Grant CE, Noble WS. The MEME suite. Nucleic Acids Res. 2015;43:W39-49.

16. Ma Q, Zhang H, Mao X, Zhou C, Liu B, Chen X, et al. DMINDA: an integrated web server for DNA motif identification and analyses. Nucleic Acids Res. 2014;42:W12-9.

17. Romero DA, Hasan AH, Lin Y-f, Kime L, Ruiz-Larrabeiti O, Urem M, et al. A comparison of key aspects of gene regulation in Streptomyces coelicolor and Escherichia coli using nucleotide-resolution transcription maps produced in parallel by global and differential RNA sequencing. Mol Microbiol. 2014;94(5):963-87.

18. Vockenhuber MP, Sharma CM, Statt MG, Schmidt D, Xu Z, Dietrich S, et al. Deep sequencing-based identification of small non-coding RNAs in Streptomyces coelicolor. RNA Biol. 2011;8(3):468-77.

19. Sass AM, Schmerk C, Agnoli K, Norville PJ, Eberl L, Valvano MA, et al. The unexpected discovery of a novel low-oxygen-activated locus for the anoxic persistence of Burkholderia cenocepacia. ISME J. 2013;7(8):1568-81.

20. Breaker RR. Riboswitches and the RNA world. Cold Spring Harb Perspect Biol. 2012;4(2):a003566

21. Storz G, Vogel J, Wassarman KM. Regulation by small RNAs in bacteria: Expanding frontiers. Mol Cell. 2011;43(6):880-91.

22. Milon P, Rodnina MV. Kinetic control of translation initiation in bacteria. Crit Rev Biochem Mol Biol. 2012;47(4):334-48.

23. Zheng X, Hu G-Q, She Z-S, Zhu H. Leaderless genes in bacteria: clue to the evolution of translation initiation mechanisms in prokaryotes. BMC Genomics. 2011;12(1):361.

24. Huang Q, Cheng X, Cheung MK, Kiselev SS, Ozoline ON, Kwan HS. Highdensity transcriptional initiation signals underline genomic islands in bacteria. PLOS ONE. 2012;7(3):e33759.

25. Wade JT, Grainger DC. Pervasive transcription: illuminating the dark matter of bacterial transcriptomes. Nat Rev Microbiol. 2014;12(9):647-53.

26. Parsons YN, Banasko R, Detsika MG, Duangsonk K, Rainbow L, Hart CA, et al. Suppression-subtractive hybridisation reveals variations in gene distribution amongst the Burkholderia cepacia complex, including the presence in some strains of a genomic island containing putative polysaccharide production genes. Arch Microbiol. 2003;179(3):214-23.

27. Dunbar J, Cohn J, Wall M. Consistency of gene starts among Burkholderia genomes. BMC Genomics. 2011;12(1):125.

28. Lybecker M, Bilusic I, Raghavan R. Pervasive transcription: detecting functional RNAs in bacteria. Transcription. 2014;5(4):e944039.

29. Thomason MK, Storz G. Bacterial antisense RNAs: How many are there, and what are they doing? Annu Rev Genet. 2010;44(1):167-88.

30. Peeters E, Sass A, Mahenthiralingam E, Nelis H, Coenye T. Transcriptional response of Burkholderia cenocepacia $\mathrm{J} 2315$ sessile cells to treatments with high doses of hydrogen peroxide and sodium hypochlorite. BMC Genomics. 2010;11(1):90.

31. Freeman ZN, Dorus S, Waterfield NR. The KdpD/KdpE two-component system: Integrating $\mathrm{K}^{+}$homeostasis and virulence. PLoS Pathog. 2013;9(3):e1003201.

32. Salvetti S, Faegri $K$, Ghelardi E, Kolsto AB, Senesi S. Global gene expression profile for swarming Bacillus cereus bacteria. Appl Environ Microbiol. 2011;77(15):5149-56.

33. Kinsinger RF, Kearns DB, Hale M, Fall R. Genetic requirements for potassium ion-dependent colony spreading in Bacillus subtilis. J Bacteriol. 2005;187(24):8462-9.

34. Beenken KE, Dunman PM, McAleese F, Macapagal D, Murphy E, Projan SJ, et al. Global gene expression in Staphylococcus aureus biofilms. J Bacteriol. 2004;186(14):4665-84.

35. Gottesman S, Storz G. Bacterial small RNA regulators: Versatile roles and rapidly evolving variations. Cold Spring Harb Perspect Biol. 2010;3(12):a003798.

36. Yoder-Himes DR, Chain PSG, Zhu Y, Wurtzel O, Rubin EM, Tiedje JM, et al. Mapping the Burkholderia cenocepacia niche response via high-throughput sequencing. P Natl Acad Sci USA. 2009;106(10):3976-81.

37. Weinberg Z, Wang J, Bogue J, Yang J, Corbino K, Moy R, et al. Comparative genomics reveals 104 candidate structured RNAs from bacteria, archaea, and their metagenomes. Genome Biol. 2010;11(3):R31.

38. Kimelman A, Levy A, Sberro H, Kidron S, Leavitt A, Amitai G, et al. A vast collection of microbial genes that are toxic to bacteria. Genome Res. 2012;22(4):802-9. 
39. Burge SW, Daub J, Eberhardt R, Tate J, Barquist L, Nawrocki EP, et al. Rfam 11.0: 10 years of RNA families. Nucleic Acids Res. 2013;41:D226-32.

40. Ramos CG, Grilo AM, da Costa PJP, Leitão JH. Experimental identification of small non-coding regulatory RNAs in the opportunistic human pathogen Burkholderia cenocepacia J2315. Genomics. 2013;101 (2):139-48.

41. Coenye T, Van Acker H, Peeters E, Sass A, Buroni S, Riccardi G, et al. Molecular mechanisms of chlorhexidine tolerance in Burkholderia cenocepacia biofilms. Antimicrob Agents Chemoth. 2011;55(5):1912-9.

42. Drevinek P, Holden MTG, Ge Z, Jones AM, Ketchell I, Gill RT, et al. Gene expression changes linked to antimicrobial resistance, oxidative stress, iron depletion and retained motility are observed when Burkholderia cenocepacia grows in cystic fibrosis sputum. BMC Infect Dis. 2008;8:121.

43. Coenye T, Drevinek P, Mahenthiralingam E, Shah SA, Gill RT, Vandamme P, et al. Identification of putative noncoding RNA genes in the Burkholderia cenocepacia J2315 genome. FEMS Microbiol Lett. 2007;276(1):83-92.

44. Vogel J, Luisi BF. Hfq and its constellation of RNA. Nat Rev Microbiol. 2011;9(8):578-89.

45. Van Acker H, De Canck E, Van Nieuwerburgh F, Sass A, Deforce D, Nelis HJ, et al. The BCESM genomic region contains a regulator involved in quorum sensing and persistence in Burkholderia cenocepacia J2315. Future Microbiol. 2014;9(7):845-60.

46. Sass A, Marchbank A, Tullis E, LiPuma JJ, Mahenthiralingam E. Spontaneous and evolutionary changes in the antibiotic resistance of Burkholderia cenocepacia observed by global gene expression analysis. BMC Genomics. 2011;12:373

47. Förstner KU, Vogel J, Sharma CM. READemption-a tool for the computational analysis of deep-sequencing-based transcriptome data. Bioinformatics. 2014;30(23):3421-3.

48. Hoffmann S, Otto C, Kurtz S, Sharma CM, Khaitovich P, Vogel J, et al. Fast mapping of short sequences with mismatches, insertions and deletions using index structures. PLoS Comput Biol. 2009;5(9):e1000502.

49. Amman F, Wolfinger M, Lorenz R, Hofacker I, Stadler P, Findeiß S. TSSAR: TSS annotation regime for dRNA-seq data. BMC Bioinformatics. 2014;15(1):89.

50. Van Acker H, Sass A, Bazzini S, De Roy K, Udine C, Messiaen T, et al. Biofilmgrown Burkholderia cepacia complex cells survive antibiotic treatment by avoiding production of reactive oxygen species. PLoS ONE. 2013;8(3):e58943.

51. Nicol JW, Helt GA, Blanchard SG, Raja A, Loraine AE. The Integrated Genome Browser: free software for distribution and exploration of genome-scale datasets. Bioinformatics. 2009;25(20):2730-1.

52. Altschul SF, Madden TL, Schäffer AA, Zhang J, Zhang Z, Miller W, et al. Gapped BLAST and PSI-BLAST: A new generation of protein database search programs. Nucleic Acids Res. 1997;25(17):3389-402.

53. Ermolaeva MD, Khalak HG, White O, Smith HO, Salzberg SL. Prediction of transcription terminators in bacterial genomes. J Mol Biol. 2000;301(1):27-33.

54. da Huang W, Sherman BT, Lempicki RA. Systematic and integrative analysis of large gene lists using DAVID bioinformatics resources. Nat Protoc. 2009;4(1):44-57.

55. Hyatt D, Chen GL, Locascio PF, Land ML, Larimer FW, Hauser L. Prodigal: prokaryotic gene recognition and translation initiation site identification. BMC Bioinformatics. 2010;11(3):119.

56. Winsor GL, Khaira B, Van Rossum T, Lo R, Whiteside MD, Brinkman FS. The Burkholderia Genome Database: facilitating flexible queries and comparative analyses. Bioinformatics. 2008;24(23):2803-4.

\section{Submit your next manuscript to BioMed Central and take full advantage of:}

- Convenient online submission

- Thorough peer review

- No space constraints or color figure charges

- Immediate publication on acceptance

- Inclusion in PubMed, CAS, Scopus and Google Scholar

- Research which is freely available for redistribution 